\title{
Molecular Assessment of Scutellaria barbata D. Don in the Treatment of Nasopharyngeal Carcinoma Based on Network Pharmacology and Experimental Verification
}

\author{
Hongjian Shi, ${ }^{1,2}$ Jie Liu, ${ }^{1,3}$ Jingying Fan, ${ }^{1,2}$ Lan He, ${ }^{3,4}$ Xianwen Wang, ${ }^{3,4}$ Faqing Tang, ${ }^{2,3}$ \\ Daofa Tian, ${ }^{2,3}$ and Yingchun He $\mathbb{D}^{1,2}$ \\ ${ }^{1}$ Hunan University of Chinese Medicine, Changsha 410208, China \\ ${ }^{2}$ Hunan Provincial Key Laboratory for Prevention and Treatment of Ophthalmology and Otolaryngology \\ Diseases with Chinese Medicine, Changsha 410208, China \\ ${ }^{3}$ Hunan Provincial Engineering and Technological Research Center for Prevention and Treatment of \\ Ophthalmology and Otolaryngology Diseases with Chinese Medicine and Protecting Visual Function, Changsha 410208, China \\ ${ }^{4}$ The first hospital of Hunan University of Chinese Medicine, Changsha 410208, China \\ Correspondence should be addressed to Yingchun He; heyingchun@hnucm.edu.cn
}

Received 24 August 2021; Revised 3 December 2021; Accepted 22 December 2021; Published 7 February 2022

Academic Editor: Yunbao Pan

Copyright (c) 2022 Hongjian Shi et al. This is an open access article distributed under the Creative Commons Attribution License, which permits unrestricted use, distribution, and reproduction in any medium, provided the original work is properly cited.

Objective. To predict the molecular mechanisms behind the benefits of Scutellaria barbata D. Don (S. barbata) in nasopharyngeal carcinoma (NPC) by network pharmacology and experimental verification. Methods. The active ingredients and targets of $S$. barbata were searched in the traditional Chinese medicine system pharmacology database and analysis platform, and the disease targets of NPC were obtained by searching the GeneCards database. A common target proteinprotein interaction network was constructed by STRING, and then, an active ingredients-NPC-target interaction network map was constructed by Cytoscape 3.7.2 software. The functional enrichment analyses of Gene Ontology and KEGG pathway data were carried out by R software programming. Finally, cell proliferation was assessed by CCK8, apoptosis was detected by Annexin V-FITC/PI double fluorescence staining, and protein expression was analyzed by Western blotting. Results. In this study, 29 active ingredients were found in S. barbata. Among these, the main targets for NPC were baicalein, wogonin, luteolin, and quercetin. The main molecular targets of $S$. barbata on NPC were EGFR, MYC, CASP3, CCND1, and ESR1. The main biological processes involved the binding of DNA-binding transcription factors, RNA polymerase IIspecific DNA-binding transcription factors, ubiquitin-like protein ligases, and ubiquitin-protein ligases. S. barbata mainly affects NPC through the PI3K-Akt, p53, and MAPK signaling pathways. The experimental results showed that baicalein and wogonin could inhibit proliferation and induce apoptosis of NPC cells and downregulate the expression of PI3K, AKT, and p53, the key proteins of the PI3K/AKT and p53 signaling pathway in CNE2 cells. Conclusion. Baicalein and wogonin, the main active ingredients of $S$. barbata, inhibited the proliferation and induced apoptosis of NPC cells through the PI3K/AKT and p53 signaling pathways.

\section{Introduction}

Nasopharyngeal carcinoma (NPC) is a malignant tumor of the head and neck, which originates from the epithelial tissue of nasopharyngeal mucosa. According to the data of the International Agency for Research on Cancer, 129079 cases of NPC were added in 2018, and 72987 patients died [1]. NPC is sensitive to radiotherapy. However, due to its concealed location, it is difficult to distinguish symptoms from benign diseases. Most NPC patients are at middle and late stages when they are diagnosed and accompanied by cervical lymph nodes and/or distant metastasis, resulting in 
a high rate of treatment failure [2]. At present, the main treatment for NPC is radiotherapy combined with platinumbased chemotherapy. However, conventional chemotherapy drugs have great toxicity and side effects, and a single drug is prone to drug resistance. A large number of studies have shown that traditional Chinese medicine (TCM) can effectively improve the efficacy of chemotherapy, radiotherapy, targeted therapy, and immunotherapy [3]. Wang et al. retrospectively evaluated the Taiwan Cancer Registry database for patients with advanced NPC from 2007 to 2013 and found that the overall risk of death in patients using Chinese herbal medicine was 0.799 times the control group, and Scutellaria barbata D. Don (S. barbata) was found to be an effective TCM for treating NPC [4].

S. barbata is a TCM, belonging to the genus Scutellaria of the Labiatae family. Modern pharmacological studies show that $S$. barbata extract has many pharmacological activities, such as antimicrobial, anti-inflammatory, and antitumor [5]. The use of $S$. barbata in antitumor treatment and research has received more and more attention, especially for colon, breast, liver, and pancreatic cancers [6-8]. The results of clinical treatment and experimental studies show that the pharmacological effects of $S$. barbata mainly include inhibiting the proliferation, migration, and invasion of tumor cells, antitumor angiogenesis, and promoting apoptosis [9-11]. However, the material basis and mechanism of $S$. barbata in the treatment of NPC remain unclear.

Network pharmacology is a new strategy of multitarget drug molecular design by selecting specific signal nodes through the network analysis of biological systems in a network database and based on the theory of systems biology. In recent years, network pharmacology has been widely used in the research of TCM to provide a new tool and method for exploring their actions of mechanism and developing active ingredients [12]. This study combines network pharmacology with experimental verification to clarify the characteristics and potential molecular mechanism of S. barbata in the treatment of NPC and to provide a reference for the development of TCM and monomers to inhibit NPC.

\section{Materials and Methods}

2.1. Screening Active Ingredients of S. barbata. S. barbata was searched in the traditional Chinese medicine system pharmacology database and analysis platform (TCMSP) database (https://old.tcmsp-e.com/index.php). The active ingredients and action targets of $S$. barbata were obtained by screening oral bioavailability $(\mathrm{OB}) \geq 30 \%$ and drug-like $(\mathrm{DL}) \geq 0.18$ substances.

2.2. Screening of Disease Targets for NPC. The keyword "nasopharyngeal carcinoma" was searched through the GeneCards database (https://www.genecards.org/) to obtain the disease target of NPC.

2.3. Screening Common Targets of Active Ingredients and Diseases and Construction of the Protein-Protein Interaction Network. The disease target was intersected with the drug target by $\mathrm{R}$ software (https://www.r-project.org/), and a Venn diagram was drawn. The protein-protein interaction (PPI) network was constructed with STRING plug (https:// string-db.org/). The protein type was set to "Homo sapiens," and other parameters were set to default to obtain the PPI network. The frequency of common protein targets was obtained by $\mathrm{R}$ software.

2.4. Drug Active Ingredients-NPC-Target Interaction Network. The interaction network between the active ingredients of S. barbata and disease targets was constructed by Cytoscape 3.7.2 software, and an interaction network diagram of drug active ingredients-NPC-targets was drawn to explore the potential mechanism of S. barbata in the treatment of NPC.

2.5. Biological Function Analysis of Gene Ontology (GO) and KEGG Pathway Enrichment Analysis. The common target sites of $S$. barbata active ingredients and NPC were analyzed by GO and KEGG pathway enrichment analysis with $\mathrm{R}$ software (https://www.r-project.org/). GO analysis was mainly used to describe the functions of gene products, including cellular, molecular, and biological functions. KEGG pathway enrichment analysis was used to analyze core pathways and explore the possible biological function and signaling pathway mechanisms in the treatment of NPC.

2.6. Experimental Verification. NPC CNE2 and 5-8F cells were treated with different concentrations of baicalein and wogonin, respectively.. The cell proliferation was assessed by the CCK8 method. The apoptosis rate was determined by Annexin V-FITC/PI double fluorescence staining, and the expression level of key proteins of the PI3K/AKT and p53 signaling pathway was analyzed by Western blotting.

2.7. Statistical Analysis. The data were processed by SPSS 26.0 statistical software and presented as the mean\pm standard deviation if they obeyed normal distribution. One way ANOVA was used to compare the measurement data between single-factor design and multiple groups. The least significant difference method was used for multiple comparisons, and the Games-Howell test was used to test the variance. The results analysis map was made by GraphPad Prism 8.0 software.

\section{Results}

3.1. Screening the Active Ingredients of S. barbata. The active ingredients of $S$. barbata were searched in the TCMSP database, and a total of 29 active ingredients were obtained. The results are given in Table 1 .

3.2. Screening of Disease Targets for NPC. After searching the GeneCards database and setting a relevance score at $\geq 5$ for preliminary screening, 722 therapeutic targets of NPC were obtained. 
TABLE 1: Main active ingredients of $S$. barbata.

\begin{tabular}{|c|c|c|c|}
\hline ID & Active ingredients & OB (\%) & $\mathrm{DL}$ \\
\hline MOL001040 & (2R)-5,7-Dihydroxy-2-(4-hydroxyphenyl) chroman-4-one & 42.36 & 0.21 \\
\hline MOL012245 & $5,7,4^{\prime}$-Trihydroxy-6-methoxyflavanone & 36.63 & 0.27 \\
\hline MOL012246 & $5,7,4^{\prime}$-Trihydroxy-8-methoxyflavanone & 74.24 & 0.26 \\
\hline MOL012248 & 5-Hydroxy-7,8-dimethoxy-2-(4-methoxyphenyl) chromone & 65.82 & 0.33 \\
\hline MOL012250 & 7-Hydroxy-5,8-dimethoxy-2-phenyl-chromone & 43.72 & 0.25 \\
\hline MOL012251 & Chrysin-5-methylether & 37.27 & 0.2 \\
\hline MOL012252 & 9,19-Cyclolanost-24-en-3-ol & 38.69 & 0.78 \\
\hline MOL002776 & Baicalein & 40.12 & 0.75 \\
\hline MOL012254 & Campesterol & 37.58 & 0.71 \\
\hline MOL000953 & CLR & 37.87 & 0.68 \\
\hline MOL000358 & Beta-sitosterol & 36.91 & 0.75 \\
\hline MOL012266 & Rivularin & 37.94 & 0.37 \\
\hline MOL001973 & Sitosteryl acetate & 40.39 & 0.85 \\
\hline MOL012269 & Stigmasta-5,22-dien-3-ol-acetate & 46.44 & 0.86 \\
\hline MOL012270 & Stigmastan-3,5,22-triene & 45.03 & 0.71 \\
\hline MOL000449 & Stigmasterol & 43.83 & 0.76 \\
\hline MOL000173 & Wogonin & 30.68 & 0.23 \\
\hline MOL001735 & Dinatin & 30.97 & 0.27 \\
\hline MOL001755 & 24-Ethylcholest-4-en-3-one & 36.08 & 0.76 \\
\hline MOL002714 & Baicalein & 33.52 & 0.21 \\
\hline MOL002719 & 6-Hydroxynaringenin & 33.23 & 0.24 \\
\hline MOL002915 & Salvigenin & 49.07 & 0.33 \\
\hline MOL000351 & Rhamnazin & 47.14 & 0.34 \\
\hline MOL000359 & Sitosterol & 36.91 & 0.75 \\
\hline MOL005190 & Eriodictyol & 71.79 & 0.24 \\
\hline MOL005869 & Daucostero_qt & 36.91 & 0.75 \\
\hline MOL000006 & Luteolin & 36.16 & 0.25 \\
\hline MOL008206 & Moslosooflavone & 44.09 & 0.25 \\
\hline MOL000098 & Quercetin & 46.43 & 0.28 \\
\hline
\end{tabular}

\subsection{Common Target Screening and Interactive Network} Construction. The therapeutic targets of NPC and the action targets of the active ingredients of $S$. barbata were screened by $\mathrm{R}$ software, and 52 common targets were obtained (Figure 1). The 52 common targets were entered into the STRING data platform to build the PPI network (Figure 2). The frequency of the occurrence of each protein target was calculated by $\mathrm{R}$ software. The most frequent protein targets were EGFR, MYC, CASP3, CCND1, and ESR1, and these could be the main potential targets for S. barbata in the treatment of NPC (Figure 3).

3.4. Construction of a Drug Active Ingredients-NPC-Target Interaction Network. The network diagram of the drug active ingredients-NPC-target interaction network (Figure 4) was drawn by Cytoscape 3.7.2 software. Yellow represents the drug $S$. barbata, red represents the disease NPC, purple represents the active ingredients of $S$. barbata, and rose-red represents the common target of the active ingredients of the drug and NPC. The results indicate that the main active ingredients of $S$. barbata on the common target were baicalein, wogonin, luteolin, and quercetin.

3.5. GO Analysis and Core Pathway Screening of S. barbata in the Treatment of NPC. The bar chart (Figure 5) and bubble chart (Figure 6) were obtained by GO analysis of the above common targets by $\mathrm{R}$ software. It was found that the

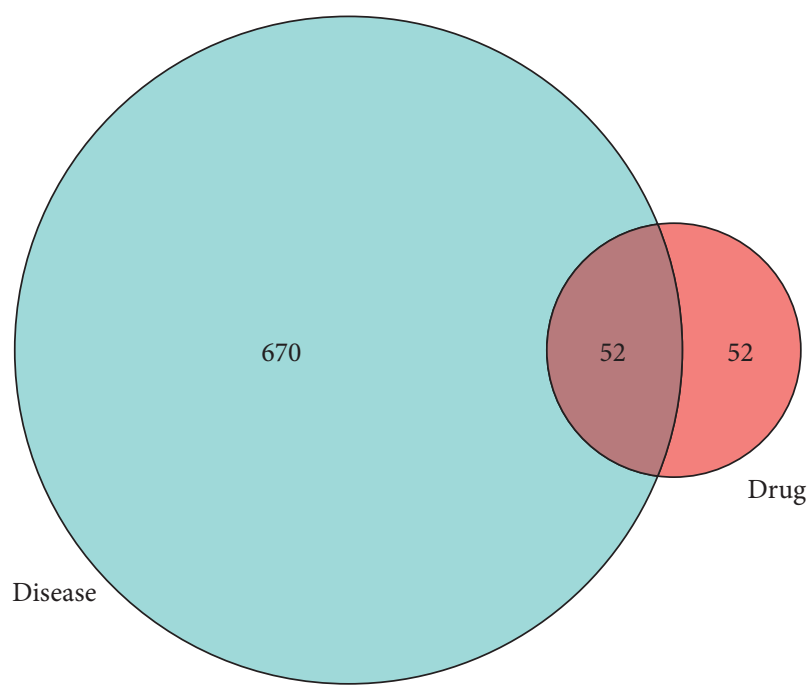

FIgURE 1: Venn diagram of the action targets of active ingredients of $S$. barbata and the therapeutic targets of NPC.

biological process of $S$. barbata acting on NPC targets mainly involved DNA-binding transcription factor binding, RNA polymerase II-specific DNA-binding transcription factor binding, ubiquitin-like protein ligase binding, and ubiquitin protein ligase binding.

Figure 7 shows the results of the KEGG pathway enrichment analysis of $S$. barbata acting on NPC targets. The main 


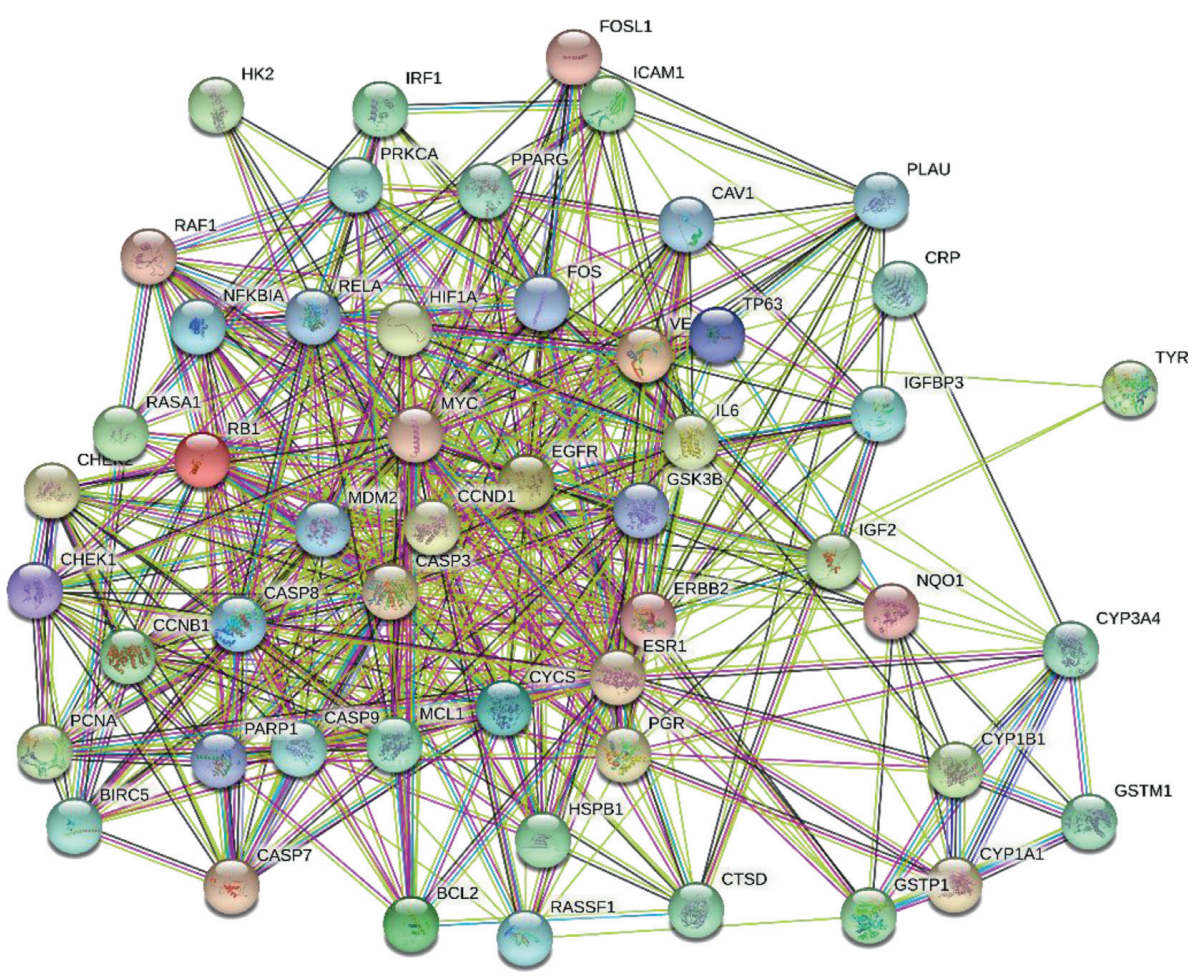

FIgURE 2: The protein-protein interaction (PPI) network.

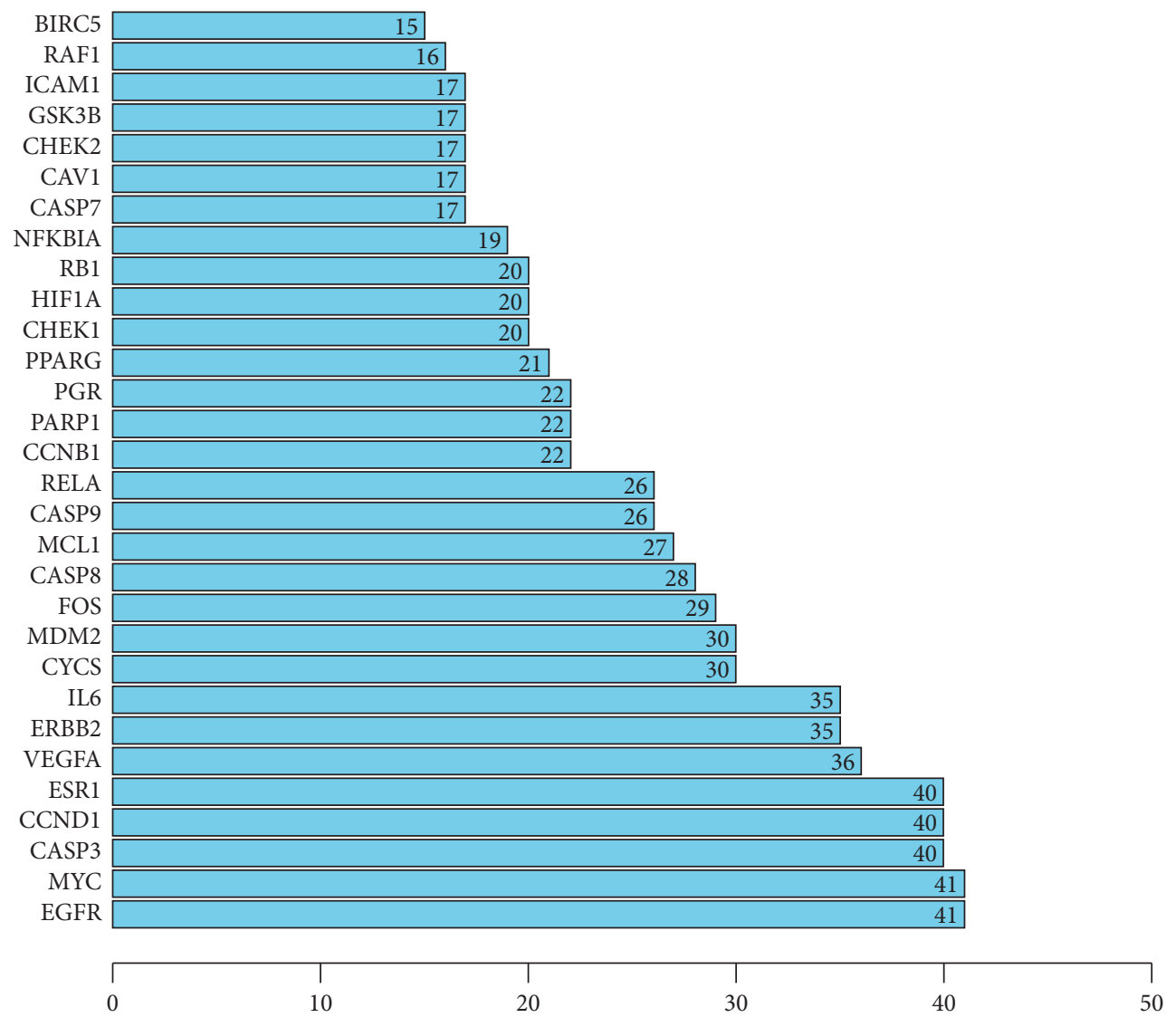

FIGURE 3: Frequency of common protein targets. 


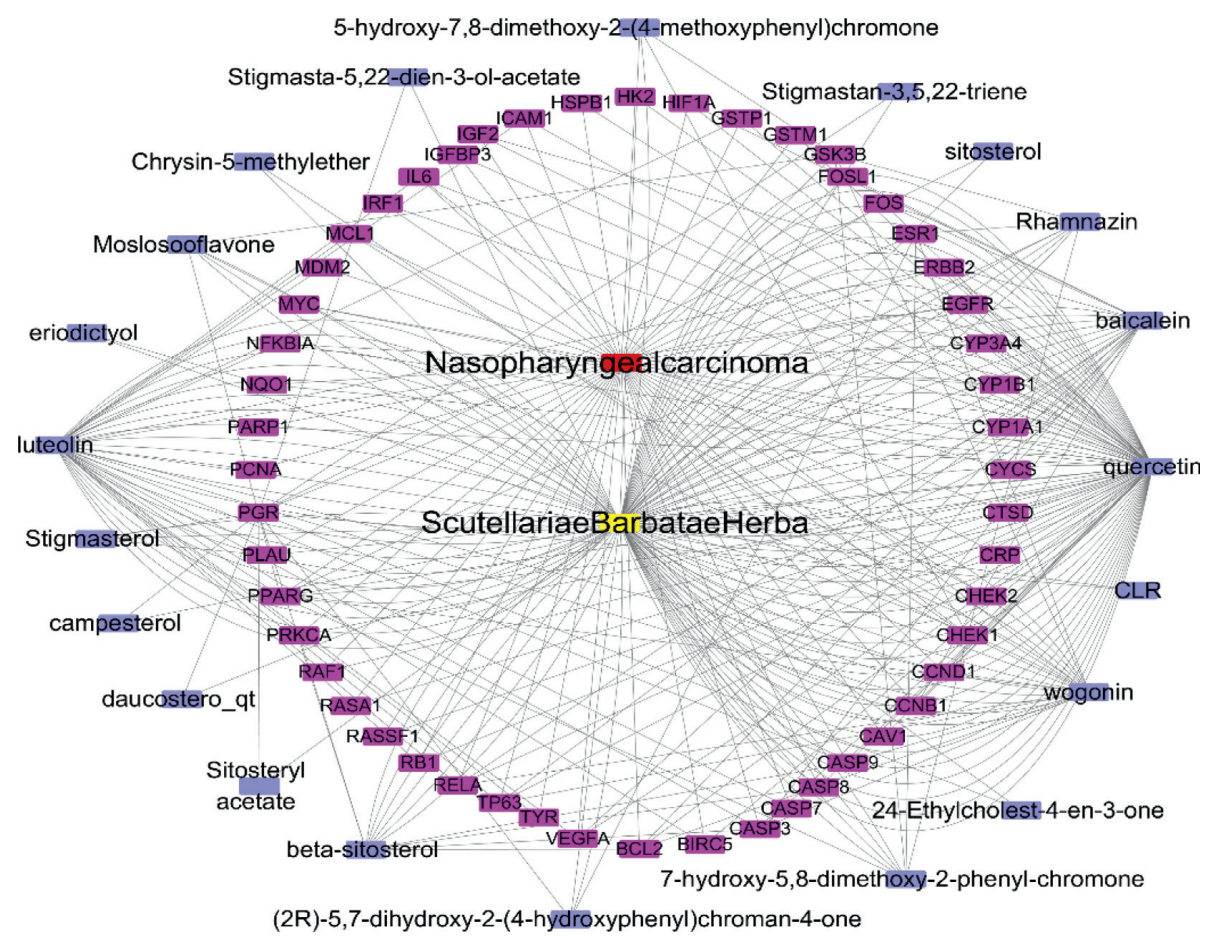

FIgURE 4: Active ingredients-NPC-target interaction network.

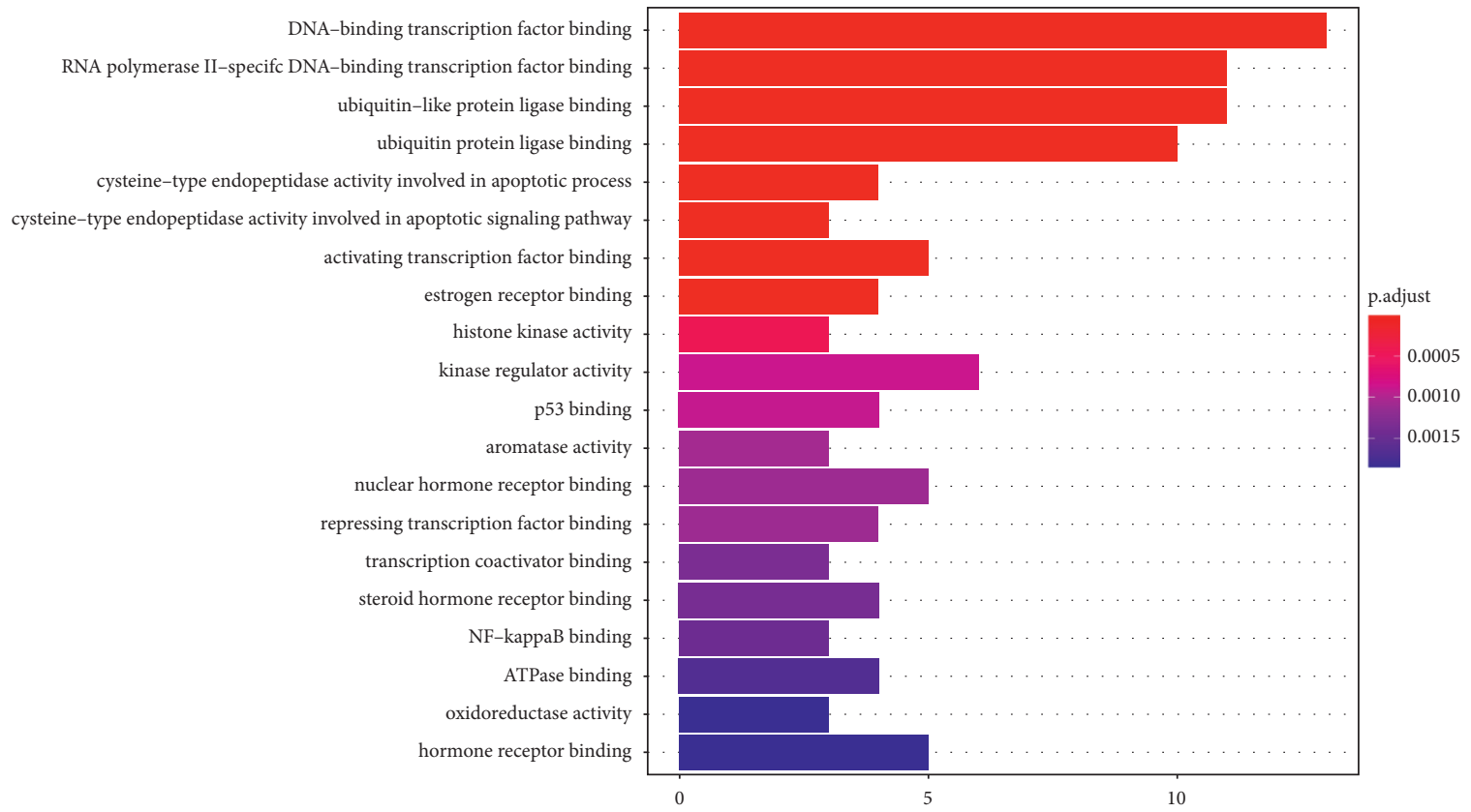

Figure 5: GO analysis bar chart.

signaling pathways with the number of enrichment targets $\geq 10$ were PI3K-Akt, MAPK, and p53 signaling pathways (Table 2).

\subsection{Experimental Results}

3.6.1. Baicalein and Wogonin Inhibit the Proliferation of NPC Cells. CCK8 results showed that baicalein significantly inhibited the proliferation of CNE2 and 5-8F cells after 24,
36, and 48 hours (Figure 8). Similarly, wogonin can inhibit the proliferation of CNE2 and 5-8F cells (Figure 9).

3.6.2. Baicalein and Wogonin Induce Apoptosis in NPC Cells. When CNE2 and 5-8F cells were treated with baicalein and wogonin, respectively, the apoptosis rate of CNE2 and 5-8F cells was significantly increased (Figure 10). 


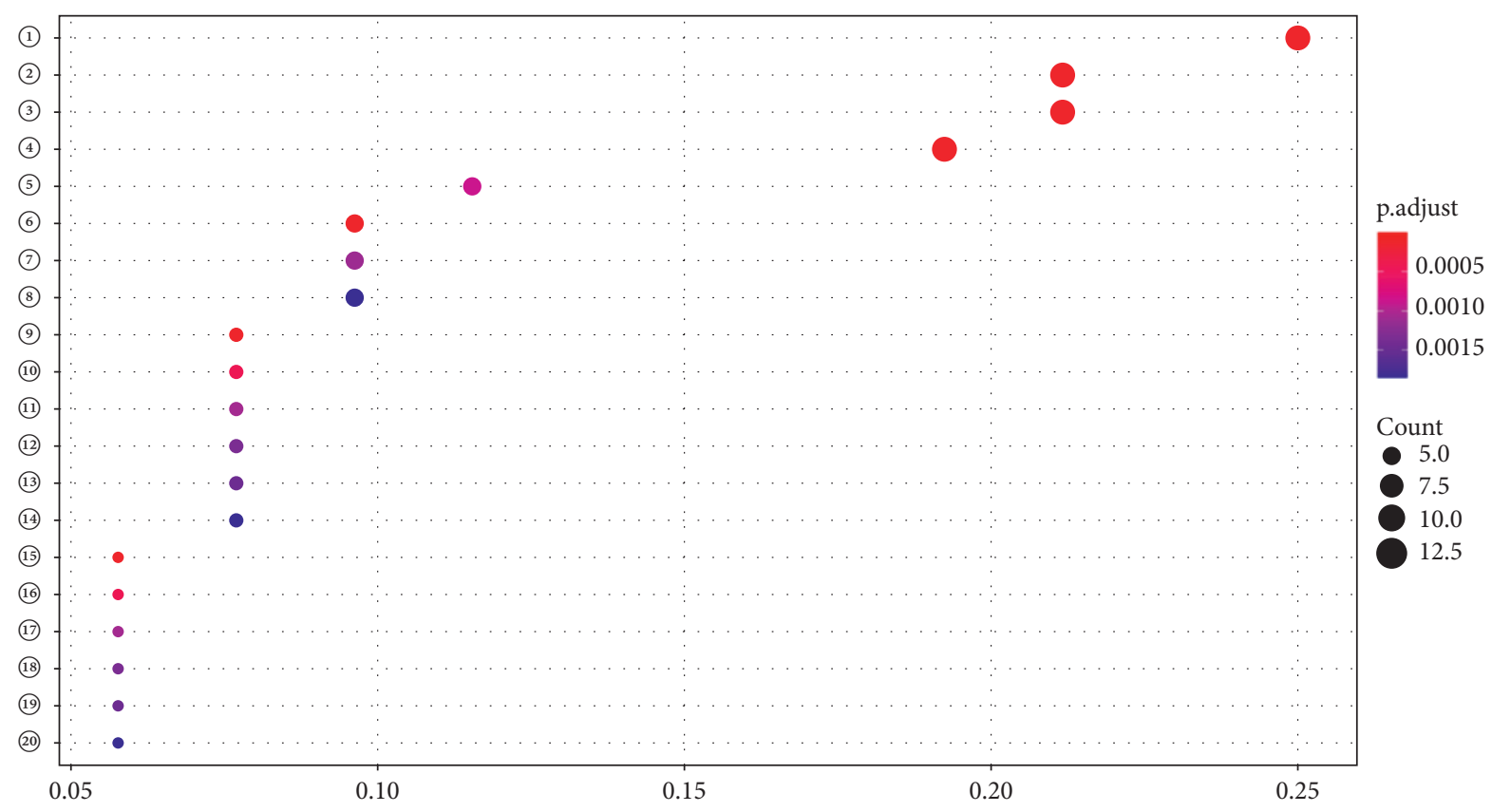

FIGURE 6: GO analysis bubble chart. The node size represents the number of enrichment targets; the color of the node from red to dark blue indicates that the $P$ value is small to large, so the larger the red node, the more significant the signaling pathway, indicating that the signaling pathway is more important. (1) DNA-binding transcription factor binding; (2) RNA polymerase II-specific DNA-binding transcription factor binding; (3) ubiquitin-like protein ligase binding; (4) ubiquitin protein ligase binding; (5) kinase regulator activity; (6) activating transcription factor binding; (7) nuclear hormone receptor binding; (8) hormone receptor binding; (9) cysteine-type endopeptidase activity involved in the apoptotic process; (10) estrogen receptor binding; (11) p53 binding; (12) repressing transcription factor binding; (13) steroid hormone receptor binding; (14) ATPase binding; (15) cysteine-type endopeptidase activity involved in the apoptotic signaling pathway; (16) histone kinase activity; (17) aromatase activity; (18) transcription coactivator binding; (19) NF-kappaB binding; (20) oxidoreductase activity.

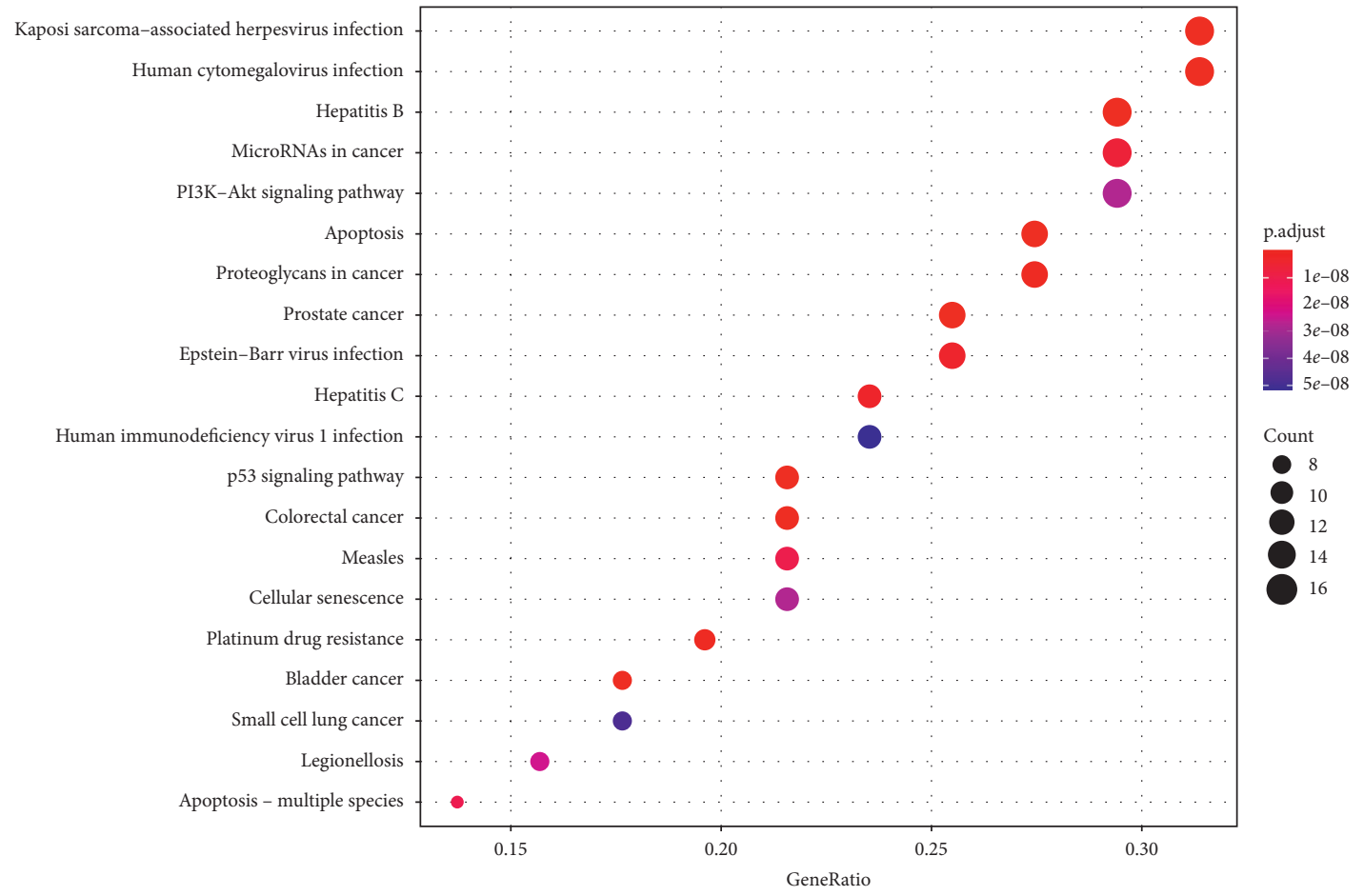

FIGURE 7: Results of KEGG enrichment analysis. 
TABLE 2: The signaling pathway of KEGG enrichment target number $\geq 10$.

\begin{tabular}{|c|c|c|c|}
\hline ID & Pathway & Genes & $\begin{array}{l}\text { Number of } \\
\text { genes }\end{array}$ \\
\hline hsa05167 & $\begin{array}{l}\text { Kaposi sarcoma-associated } \\
\text { herpesvirus infection }\end{array}$ & $\begin{array}{c}\text { GSK3B/CASP9/CASP3/CASP8/RELA/CCND1/IL6/VEGFA/FOS/ } \\
\text { HIF1A/CYCS/RB1/NFKBIA/ICAM1/RAF1/MYC }\end{array}$ & 16 \\
\hline hsa05163 & Human cytomegalovirus infection & $\begin{array}{c}\text { GSK3B/CASP9/CASP3/CASP8/PRKCA/RELA/CCND1/IL6/VEGFA/ } \\
\text { CYCS/EGFR/RB1/NFKBIA/MDM2/RAF1/MYC }\end{array}$ & 16 \\
\hline hsa05161 & Hepatitis B & $\begin{array}{c}\text { BCL2/CASP9/CASP3/CASP8/PRKCA/RELA/IL6/FOS/CYCS/RB1/ } \\
\text { NFKBIA/PCNA/BIRC5/RAF1/MYC }\end{array}$ & 15 \\
\hline hsa05206 & MicroRNAs in cancer & $\begin{array}{c}\text { BCL2/CASP3/PRKCA/PLAU/CCND1/TP63/MCL1/VEGFA/EGFR/ } \\
\text { MDM2/ERBB2/RAF1/MYC/CYP1B1/RASSF1 }\end{array}$ & 15 \\
\hline hsa04151 & PI3K-Akt signaling pathway & $\begin{array}{c}\text { GSK3B/BCL2/CASP9/PRKCA/RELA/CCND1/IL6/MCL1/VEGFA/ } \\
\text { IGF2/EGFR/MDM2/ERBB2/RAF1/MYC }\end{array}$ & 15 \\
\hline hsa04210 & Apoptosis & $\begin{array}{c}\text { BCL2/CASP9/CASP3/CASP8/RELA/MCL1/FOS/CYCS/NFKBIA/ } \\
\text { CASP7/BIRC5/RAF1/PARP1/CTSD }\end{array}$ & 14 \\
\hline hsa05205 & Proteoglycans in cancer & $\begin{array}{l}\text { ESR1/CASP3/PRKCA/PLAU/CCND1/VEGFA/HIF1A/IGF2/EGFR/ } \\
\text { MDM2/ERBB2/RAF1/CAV1/MYC }\end{array}$ & 14 \\
\hline hsa05215 & Prostate cancer & $\begin{array}{c}\text { GSK3B/BCL2/CASP9/PLAU/RELA/CCND1/EGFR/RB1/NFKBIA/ } \\
\text { MDM2/ERBB2/GSTP1/RAF1 }\end{array}$ & 13 \\
\hline hsa05169 & Epstein-Barr virus infection & $\begin{array}{c}\text { BCL2/CASP9/CASP3/CASP8/RELA/CCND1/IL6/CYCS/RB1/ } \\
\text { NFKBIA/MDM2/ICAM1/MYC }\end{array}$ & 13 \\
\hline hsa05160 & Hepatitis C & $\begin{array}{c}\text { GSK3B/CASP9/CASP3/CASP8/RELA/CCND1/CYCS/EGFR/RB1/ } \\
\text { NFKBIA/RAF1/MYC }\end{array}$ & 12 \\
\hline hsa05170 & $\begin{array}{c}\text { Human immunodeficiency virus } 1 \\
\text { infection }\end{array}$ & $\begin{array}{c}\text { CHEK1/BCL2/CASP9/CASP3/CASP8/PRKCA/RELA/FOS/CCNB1/ } \\
\text { CYCS/NFKBIA/RAF1 }\end{array}$ & 12 \\
\hline hsa04010 & MAPK signaling pathway & $\begin{array}{c}\text { CASP3/PRKCA/RELA/VEGFA/FOS/IGF2/EGFR/ERBB2/RAF1/MYC/ } \\
\text { HSPB1/RASA1 }\end{array}$ & 12 \\
\hline hsa04115 & p53 signaling pathway & $\begin{array}{c}\text { CHEK1/BCL2/CASP9/CASP3/CASP8/CCND1/CCNB1/CYCS/ } \\
\text { MDM2/CHEK2/IGFBP3 }\end{array}$ & 11 \\
\hline hsa05210 & Colorectal cancer & $\begin{array}{c}\text { GSK3B/BCL2/CASP9/CASP3/CCND1/FOS/CYCS/EGFR/BIRC5/ } \\
\text { RAF1/MYC }\end{array}$ & 11 \\
\hline hsa05162 & Measles & $\begin{array}{c}\text { GSK3B/BCL2/CASP9/CASP3/CASP8/RELA/CCND1/IL6/FOS/CYCS/ } \\
\text { NFKBIA }\end{array}$ & 11 \\
\hline hsa04218 & Cellular senescence & $\begin{array}{c}\text { CHEK1/RELA/CCND1/IL6/CCNB1/RB1/MDM2/RAF1/MYC/ } \\
\text { CHEK2/IGFBP3 }\end{array}$ & 11 \\
\hline hsa05225 & Hepatocellular carcinoma & $\begin{array}{c}\text { GSK3B/PRKCA/CCND1/IGF2/NQO1/EGFR/RB1/GSTP1/RAF1/ } \\
\text { MYC/GSTM1 }\end{array}$ & 11 \\
\hline hsa05166 & $\begin{array}{l}\text { Human T cell leukemia virus } 1 \\
\text { infection }\end{array}$ & $\begin{array}{l}\text { CHEK1/RELA/CCND1/IL6/FOS/FOSL1/RB1/NFKBIA/ICAM1/MYC/ } \\
\text { CHEK2 }\end{array}$ & 11 \\
\hline hsa05132 & Salmonella infection & $\begin{array}{l}\text { BCL2/CASP3/CASP8/RELA/IL6/FOS/CYCS/NFKBIA/CASP7/RAF1/ } \\
\text { MYC }\end{array}$ & 11 \\
\hline hsa05165 & Human papillomavirus infection & $\begin{array}{l}\text { GSK3B/CASP3/CASP8/RELA/CCND1/VEGFA/EGFR/RB1/MDM2/ } \\
\text { RAF1/IRF1 }\end{array}$ & 11 \\
\hline hsa05022 & $\begin{array}{l}\text { Pathways of neurodegeneration, } \\
\text { multiple diseases }\end{array}$ & $\begin{array}{l}\text { GSK3B/BCL2/CASP9/CASP3/CASP8/PRKCA/RELA/IL6/CYCS/ } \\
\text { CASP7/RAF1 }\end{array}$ & 11 \\
\hline hsa01524 & Platinum drug resistance & $\begin{array}{l}\text { BCL2/CASP9/CASP3/CASP8/CYCS/MDM2/ERBB2/BIRC5/GSTP1/ } \\
\text { GSTM1 }\end{array}$ & 10 \\
\hline hsa05224 & Breast cancer & ESR1/PGR/GSK3B/CCND1/FOS/EGFR/RB1/ERBB2/RAF1/MYC & 10 \\
\hline hsa05164 & Influenza A & $\begin{array}{l}\text { CASP9/CASP3/CASP8/PRKCA/RELA/IL6/CYCS/NFKBIA/ICAM1/ } \\
\text { RAF1 }\end{array}$ & 10 \\
\hline hsa05167 & $\begin{array}{l}\text { Kaposi sarcoma-associated } \\
\text { herpesvirus infection }\end{array}$ & $\begin{array}{c}\text { GSK3B/CASP9/CASP3/CASP8/RELA/CCND1/IL6/VEGFA/FOS/ } \\
\text { HIF1A/CYCS/RB1/NFKBIA/ICAM1/RAF1/MYC }\end{array}$ & 16 \\
\hline hsa05163 & Human cytomegalovirus infection & $\begin{array}{l}\text { GSK3B/CASP9/CASP3/CASP8/PRKCA/RELA/CCND1/IL6/VEGFA/ } \\
\text { CYCS/EGFR/RB1/NFKBIA/MDM2/RAF1/MYC }\end{array}$ & 16 \\
\hline hsa05161 & Hepatitis B & $\begin{array}{c}\text { BCL2/CASP9/CASP3/CASP8/PRKCA/RELA/IL6/FOS/CYCS/RB1/ } \\
\text { NFKBIA/PCNA/BIRC5/RAF1/MYC }\end{array}$ & 15 \\
\hline hsa05206 & MicroRNAs in cancer & $\begin{array}{c}\text { BCL2/CASP3/PRKCA/PLAU/CCND1/TP63/MCL1/VEGFA/EGFR/ } \\
\text { MDM2/ERBB2/RAF1/MYC/CYP1B1/RASSF1 }\end{array}$ & 15 \\
\hline hsa04151 & PI3K-Akt signaling pathway & $\begin{array}{l}\text { GSK3B/BCL2/CASP9/PRKCA/RELA/CCND1/IL6/MCL1/VEGFA/ } \\
\text { IGF2/EGFR/MDM2/ERBB2/RAF1/MYC }\end{array}$ & 15 \\
\hline
\end{tabular}



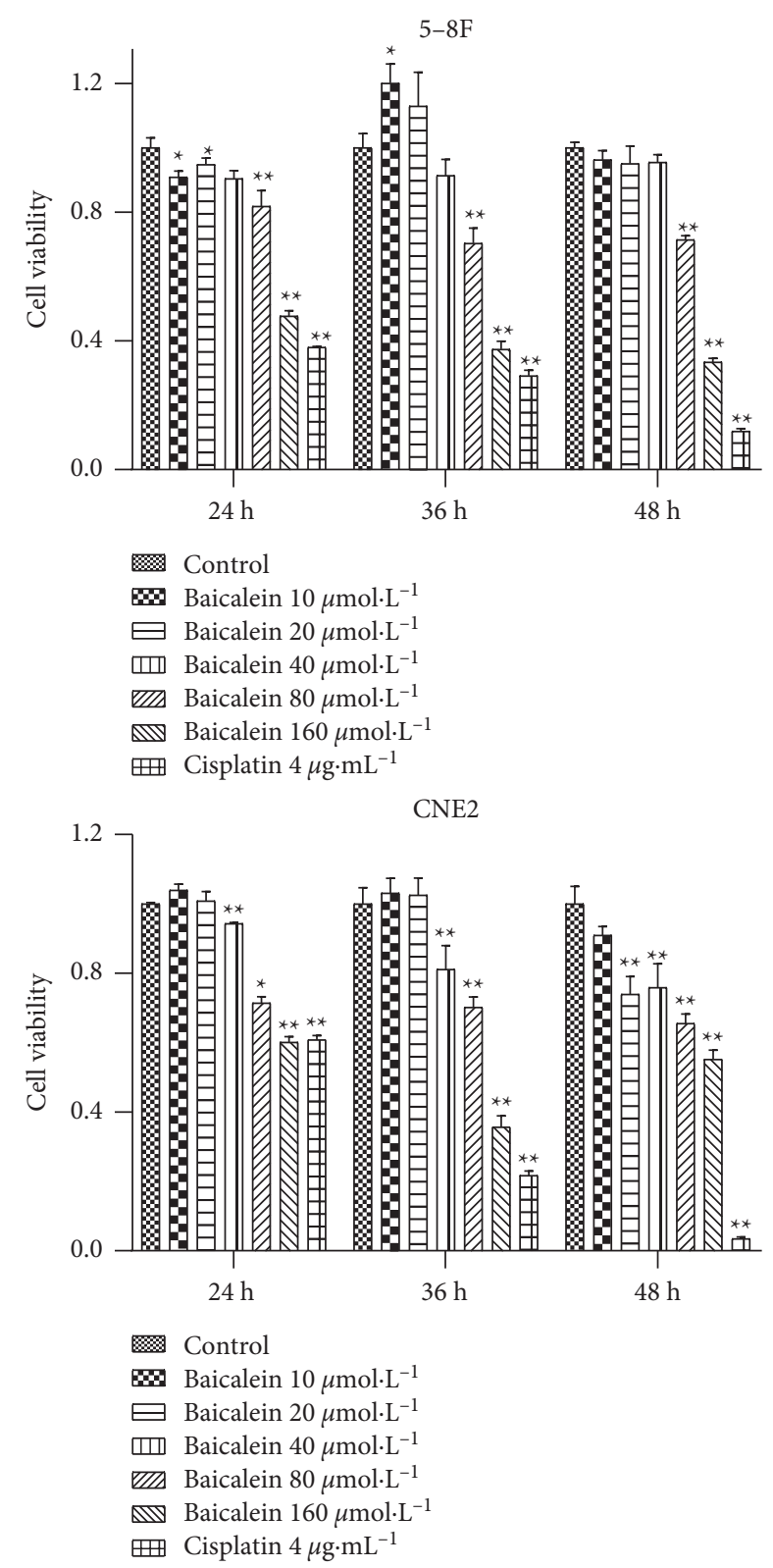

FIGURE 8: CCK8 assessment of the effect of baicalein on the proliferation of $\mathrm{CNE} 2$ and $5-8 \mathrm{~F}$ cells (vs. the control group: ${ }^{*} P<0.05$ and $\left.{ }^{* *} P<0.01\right)$.

3.6.3. Baicalein and Wogonin Inhibit the Activity of Key Proteins in the PI3K/AKT and p53 Signaling Pathway. As shown in Figure 11, baicalein and wogonin significantly decreased the expression of PI3K, AKT, and p53, the key proteins in the PI3K/AKT and p53 signaling pathway, in CNE2 cells treated with either baicalein or wogonin.

\section{Discussion}

S. barbata, also known as "Ban Zhi Lian" in China, has heatclearing and detoxifying properties (Qingre Jiedu in Chinese) [5]. According to the 2015 edition of the Chinese Pharmacopoeia, $S$. barbata can be used to treat various inflammations, edema, traumatic injury, macula, and snake bites. In recent

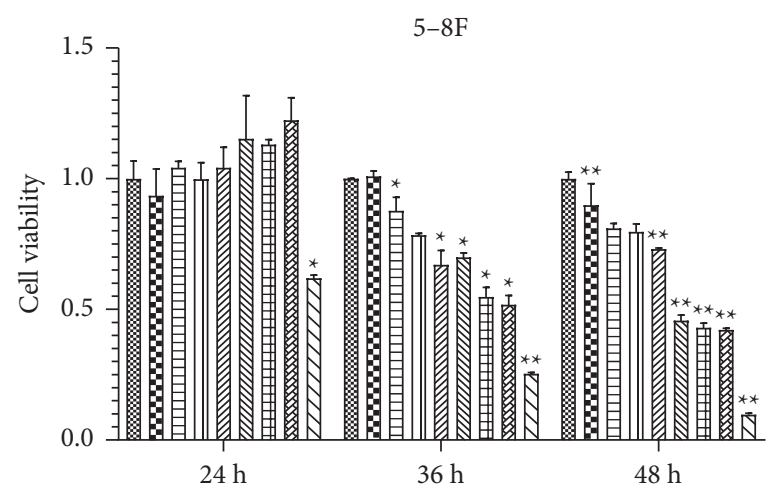

Control

$\infty$ Wogonin $2.5 \mu \mathrm{mol} \cdot \mathrm{L}^{-1}$

$\simeq$ Wogonin $5 \mu \mathrm{mol} \cdot \mathrm{L}^{-1}$

س Wogonin $10 \mu \mathrm{mol} \cdot \mathrm{L}^{-1}$

Wogonin $20 \mu \mathrm{mol} \cdot \mathrm{L}^{-1}$

Wogonin $40 \mu \mathrm{mol} \cdot \mathrm{L}^{-1}$

mogonin $80 \mu \mathrm{mol} \cdot \mathrm{L}^{-1}$

Wogonin $160 \mu \mathrm{mol} \cdot \mathrm{L}^{-1}$

Cisplatin $4 \mu \mathrm{g} \cdot \mathrm{mL}^{-1}$

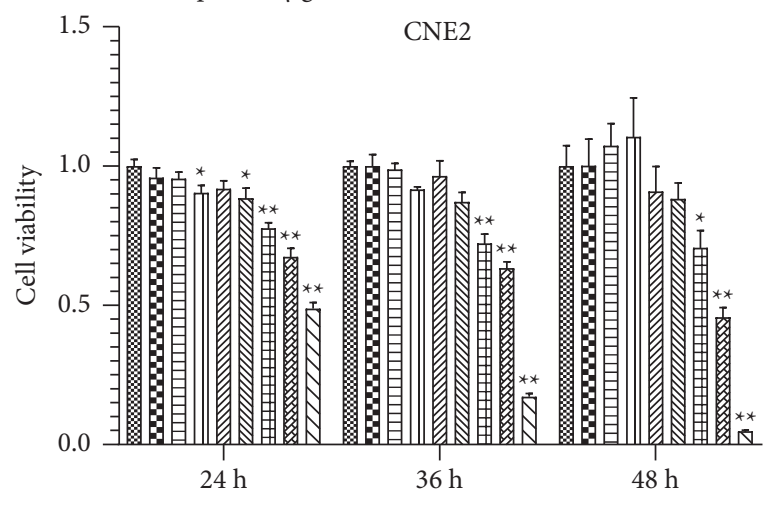

Control

$\infty$ Wogonin $2.5 \mu \mathrm{mol} \cdot \mathrm{L}^{-1}$

$\square$ Wogonin $5 \mu \mathrm{mol} \cdot \mathrm{L}^{-1}$

mogonin $10 \mu \mathrm{mol} \cdot \mathrm{L}^{-1}$

Wogonin $20 \mu \mathrm{mol} \cdot \mathrm{L}^{-1}$

Wogonin $40 \mu \mathrm{mol} \cdot \mathrm{L}^{-1}$

mogonin $80 \mu \mathrm{mol} \cdot \mathrm{L}^{-1}$

Wogonin $160 \mu \mathrm{mol} \cdot \mathrm{L}^{-1}$

$\triangle$ Cisplatin $4 \mu \mathrm{g} \cdot \mathrm{mL}^{-1}$

FIgURE 9: CCK8 assessment of the effect of wogonin on the proliferation of $\mathrm{CNE} 2$ and $5-8 \mathrm{~F}$ cells (vs. the control group: ${ }^{*} P<0.05$ and $\left.{ }^{* *} P<0.01\right)$.

years, it is often used alone or in combination with other TCM to treat various cancers [13]. For example, Yang et al. found that S. barbata combined with Hedyotis diffusa can inhibit breast cancer by interfering with the miR-200c-PDE7B/PD-L1-AKT/ MAPK axis [14]. Modern pharmacological studies have shown that $S$. barbata contains a variety of active chemical components, including flavonoids, polysaccharides, diterpenes, and porphyrins, which have good antitumor activity and can achieve an antitumor effect through different mechanisms [15].

This study uses network pharmacology to explore the molecular mechanism of S. barbata in the treatment of NPC. Through database screening, a total of 29 bioactive 

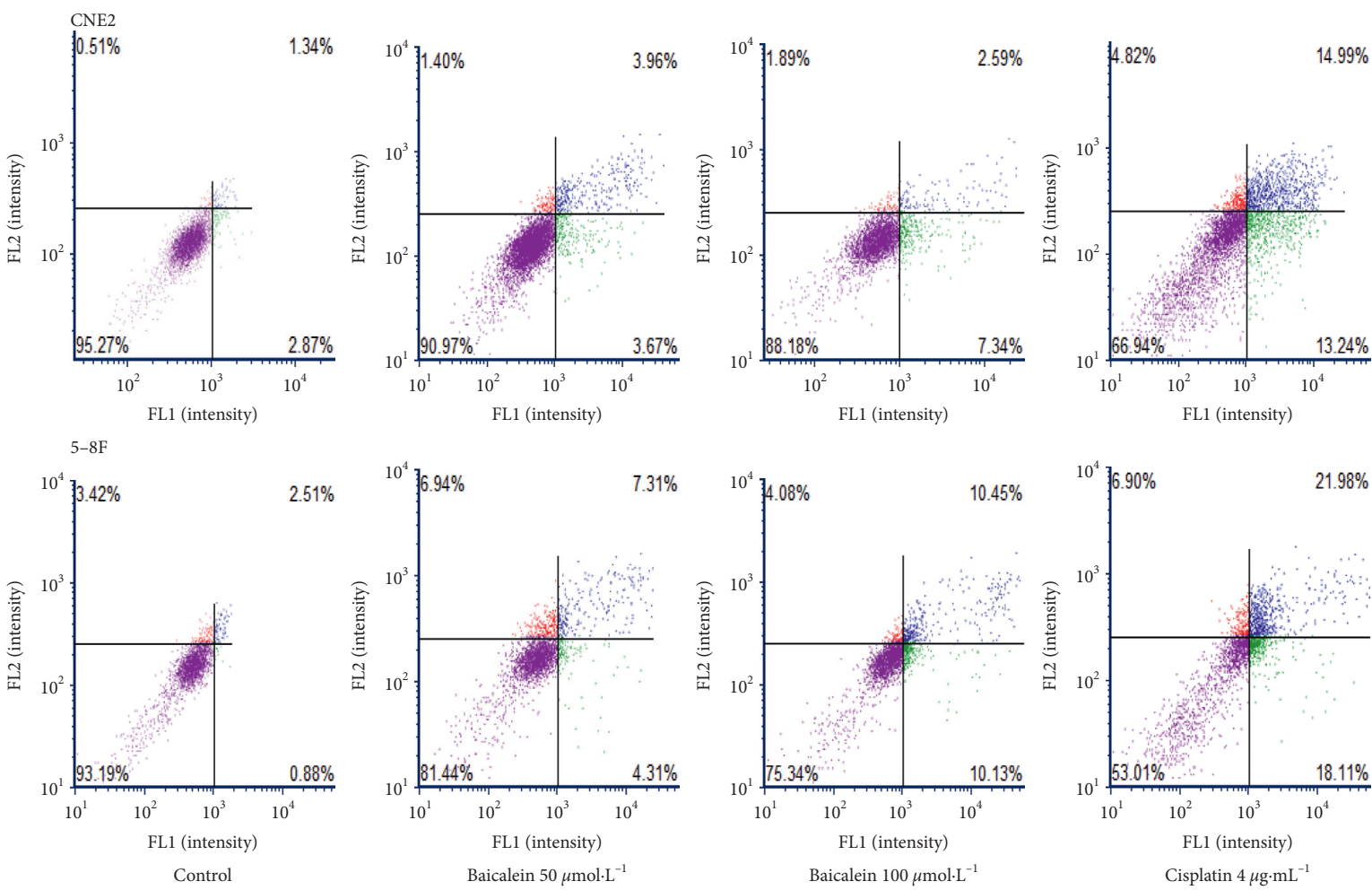

Lower left: Live

$\square$ Lower right: Apoptotic

upper right: Necrotic

$\square$ Upper left: Debris

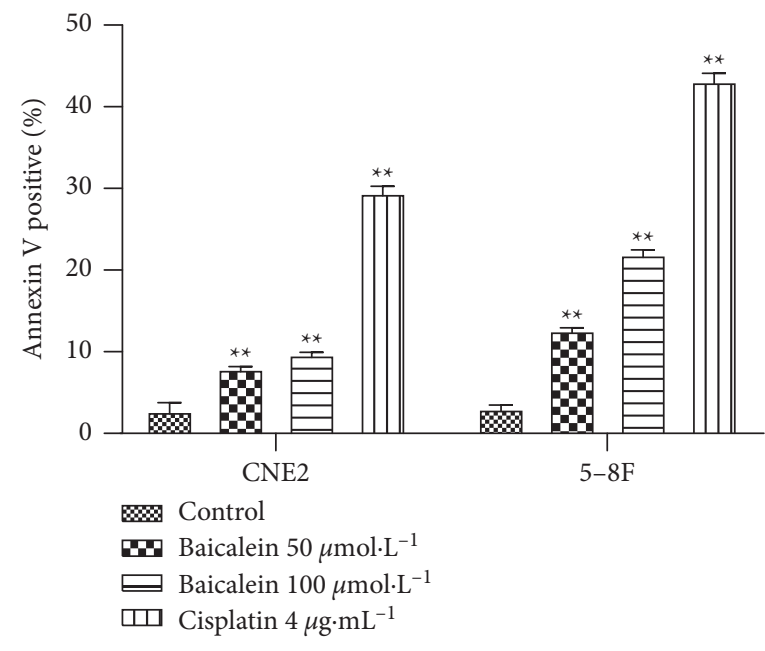

Figure 10: Continued. 

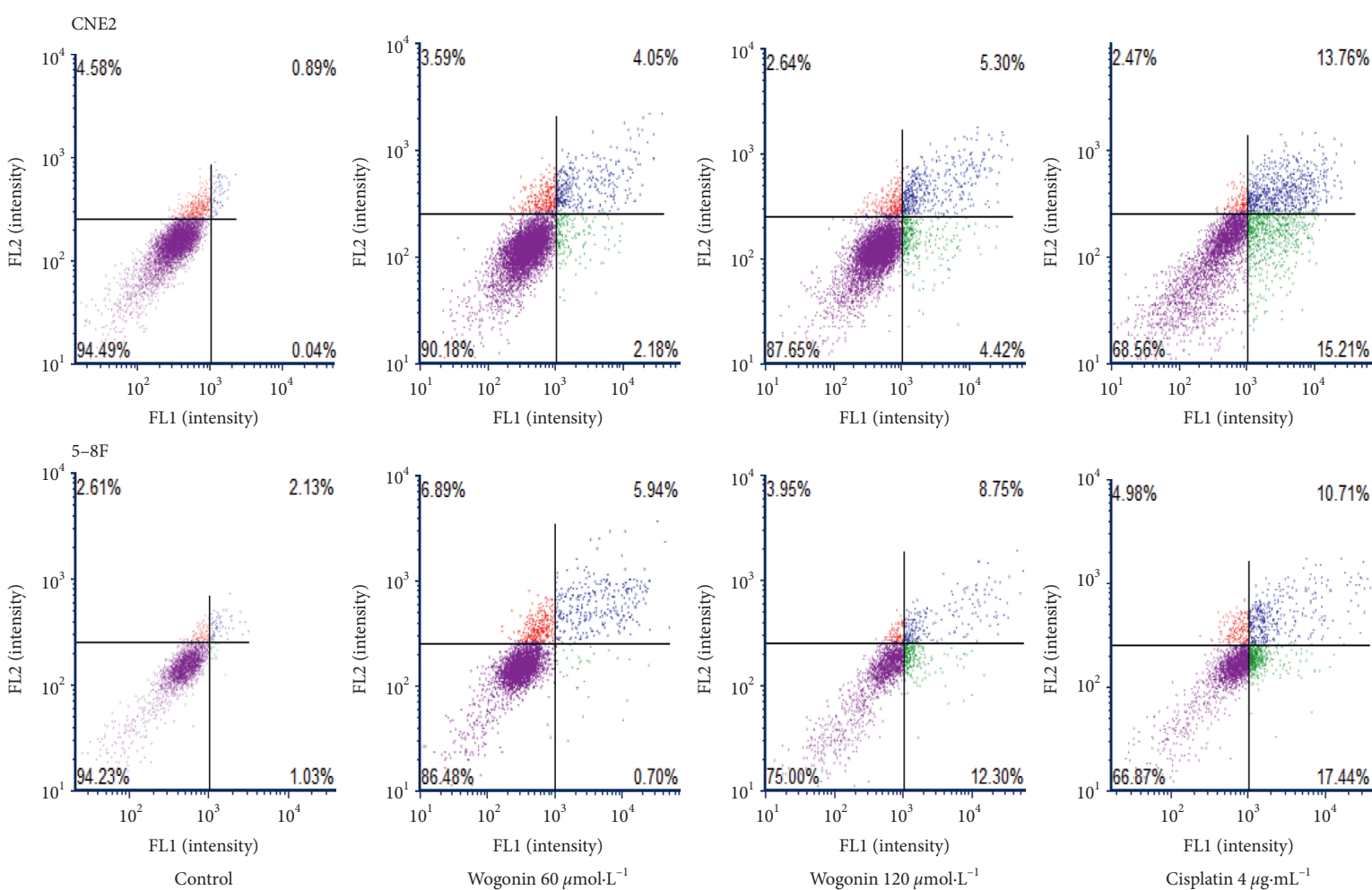

Lower left: Live

Lower right: Apoptotic

upper right: Necrotic

$\square$ Upper left: Debris

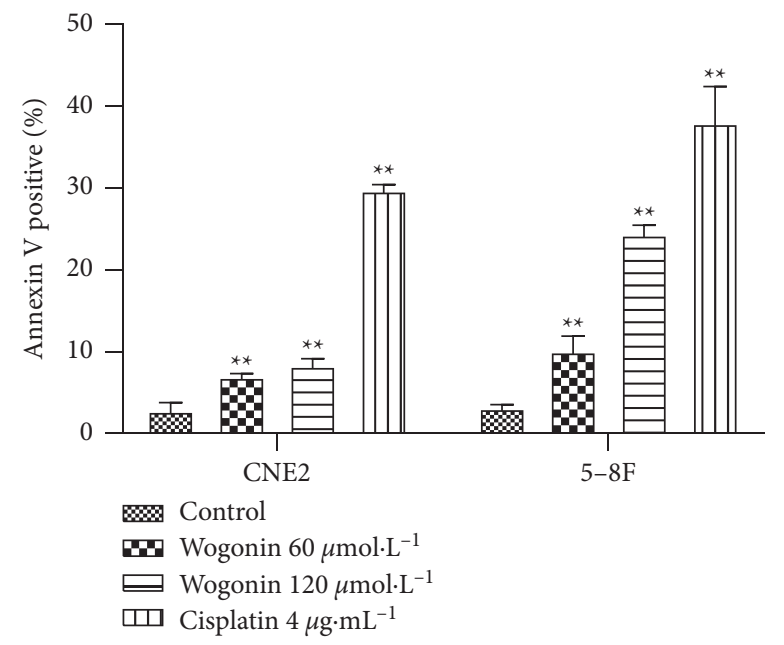

FIGURE 10: Annexin V-FITC/PI double fluorescence staining used to detect the effect of baicalein and wogonin on apoptosis rate (vs. the control group: $\left.{ }^{* *} P<0.01\right)$.

ingredients were obtained from S. barbata. From the construction of the active ingredients-target interaction network, it was found that the main active ingredients of S. barbata were baicalein, wogonin, luteolin, and quercetin. Baicalein and wogonin are flavonoids in S. barbata. Related studies have shown that baicalein can exert its antitumor effect by inducing tumor cell apoptosis and invasion, inhibiting tumor angiogenesis and scavenging free radicals
[16]. Wogonin can promote the apoptosis of tumor cells, enhance the toxicity of TNF- $\alpha$ and TRAIL to tumor cells, block the tumor cell cycle, inhibit tumor angiogenesis, and cooperate with chemotherapeutic drugs through ROS and Ca2+-mediated signaling pathways [17]. We confirmed that both baicalein and wogonin can inhibit the proliferation of NPC CNE2 and 5-8F cells and induce apoptosis by CCK8 and Annexin V-FITC/PI double fluorescence staining, 

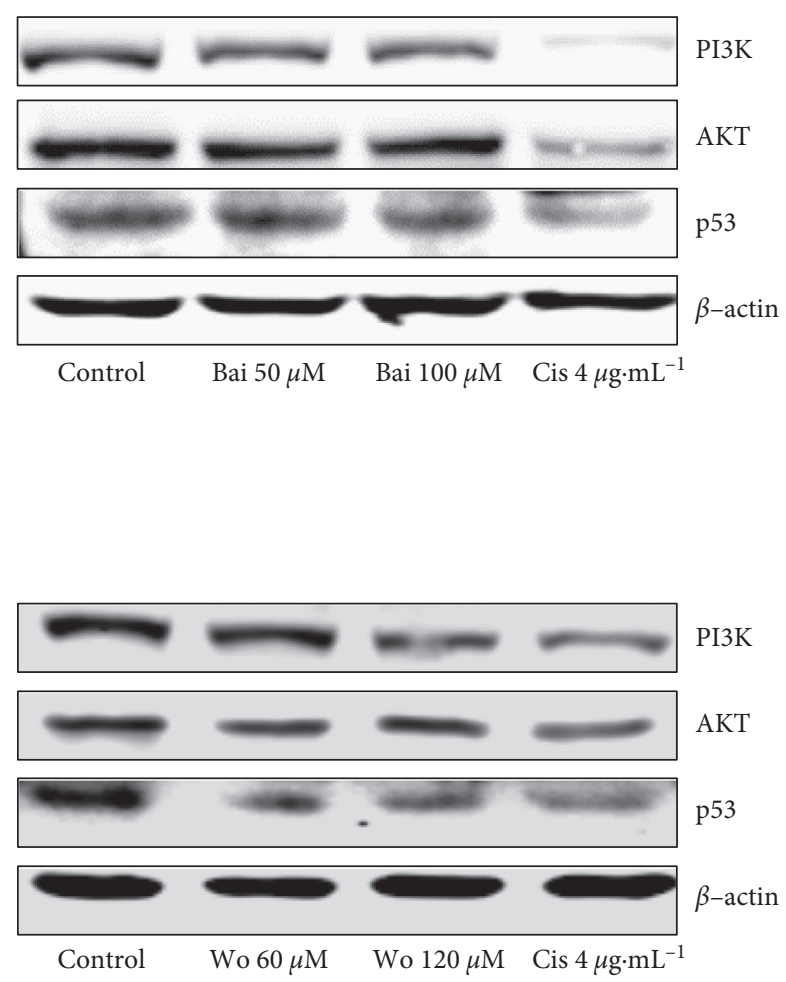

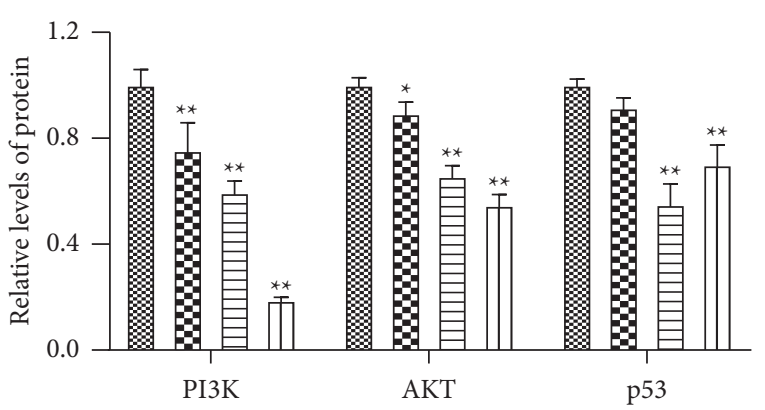

\&8) Control

Haicalein $50 \mu \mathrm{mol} \cdot \mathrm{L}^{-1}$ $\Longrightarrow$ Baicalein $100 \mu \mathrm{mol} \cdot \mathrm{L}^{-1}$ एत Cisplatin $4 \mu \mathrm{g} \cdot \mathrm{mL}^{-1}$

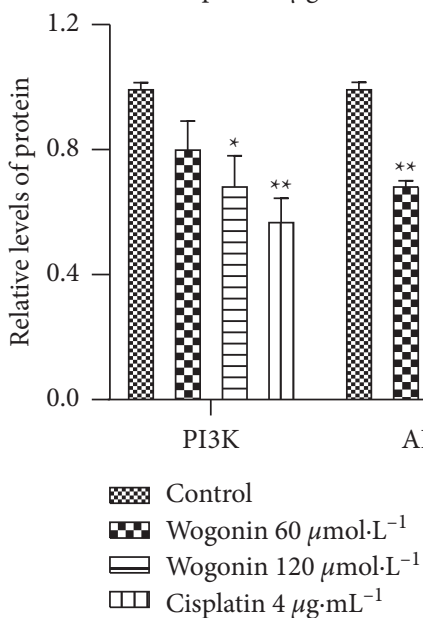

FIGURE 11: The effects of baicalein and wogonin on the expression of key proteins of the PI3K/AKT and p53 signaling pathway in CNE2 cells analyzed by Western blotting (vs. the control group: ${ }^{*} P<0.05$ and ${ }^{* *} P<0.01$ ).

which is consistent with the results of network pharmacological prediction, indicating that the method based on network pharmacology to predict the effective components of TCM is scientific and feasible.

To determine the pharmacological mechanism of $S$. barbata on NPC, we analyzed the active ingredients of S. barbata and NPC targets by using a PPI network and found that the protein targets with high frequency were EGFR, MYC, CASP3, CCND1, and ESR1. EGFR is a transmembrane receptor tyrosine kinase that regulates a variety of cellular functions, including cell proliferation, differentiation, and migration [18]. The upregulation of the EGFR signaling pathway is associated with many cancers, including glioblastoma, lung adenocarcinoma, and endometrial carcinoma [19-21]. The expression of MYC is upregulated in NPC and is related to the proliferation and metastasis of NPC cells [22]. The results of GO function analysis showed that the biological processes of $S$. barbata affecting NPC were mainly related to DNA-binding transcription factors, RNA polymerase II-specific DNA-binding transcription factors, ubiquitin-like protein ligase binding, and ubiquitin-protein ligase binding.

KEGG pathway enrichment analysis showed that S. barbata affected NPC mainly through the PI3K-Akt, p53, and MAPK signaling pathways. In an acupuncture study of core binding factor-related acute myeloid leukemia, it was found that baicalein could induce apoptosis, accompanied by p53-mediated apoptotic gene expression [23]. Wang et al. found that baicalein inhibits proliferation and induces apoptosis of bladder cancer cells by inhibiting the PI3K/AKT/ mTOR signaling pathway in vivo and in vitro [24]. It has been found that wogonin inhibits the proliferation of human colorectal cancer cells by inducing autophagy, apoptosis, and G2/M cell cycle arrest via modulating the PI3K/AKT and STAT3 signaling pathways [25]. Our results also showed that baicalein and wogonin inhibited the expressions of PI3K, $\mathrm{AKT}$, and p53, which are the key proteins in the PI3K/AKT and p53 signaling pathway in CNE2 cells, suggesting that the main active ingredients of $S$. barbata can play an anti-NPC effect through the PI3K/AKT and p53 signaling pathway.

This study systematically predicted the material basis and mechanism of $S$. barbata in the treatment of NPC. Through experiments, it was found that the main active ingredients of $S$. barbata, baicalein and wogonin, could inhibit the proliferation and induce apoptosis of NPC cells and inhibit the activity of the PI3K/AKT and p53 signaling pathway, which provides a theoretical basis for clinical intervention of NPC by S. barbata and provides evidence and references for the development of TCM and monomers in the treatment of NPC. 


\section{Data Availability}

S. barbata was searched in the TCMSP database by entering "Banzhilian". (https://old.tcmsp-e.com/index.php.). The active ingredients and action targets of $S$. barbata were obtained by screening oral bioavailability (OB) $\geq 30 \%$ and drug-like $(\mathrm{DL}) \geq 0.18$ substances. The keyword "nasopharyngeal carcinoma" was searched through the GeneCards database (https://www.genecards.org/) to obtain the disease target of NPC. The disease target was intersected with the drug target by R software (https://www.r-project.org/), and a Venn diagram was drawn. The PPI network was constructed with STRING plug (https://string-db.org/).

\section{Conflicts of Interest}

The authors declare that they have no conflicts of interest.

\section{Acknowledgments}

This research was funded by the National Natural Science Foundation of China (81874408 and 81973914), the Natural Science Foundation of Hunan Province (2020JJ5419), and the Project of Hunan Provincial Administration of Traditional Chinese Medicine (2021014 and 2021184).

\section{References}

[1] F. Bray, J. Ferlay, I. Soerjomataram, R. L. Siegel, L. A. Torre, and A. Jemal, "Global cancer statistics 2018: GLOBOCAN estimates of incidence and mortality worldwide for 36 cancers in 185 countries," CA: A Cancer Journal for Clinicians, vol. 68, no. 6, pp. 394-424, 2018.

[2] Y. M. Tian, M. Z. Liu, L. Zeng et al., "Long-term outcome and pattern of failure for patients with nasopharyngeal carcinoma treated with intensity-modulated radiotherapy," Head \& Neck, vol. 41, no. 5, pp. 1246-1252, 2019.

[3] S. Wang, S. Long, Z. Deng, and W. Wu, "Positive role of Chinese herbal medicine in cancer immune regulation," The American Journal of Chinese Medicine, vol. 48, no. 7, pp. 1577-1592, 2020.

[4] C. Y. Wang, T. C. Wang, W. M. Liang et al., "Effect of Chinese herbal medicine therapy on overall and cancer related mortality in patients with advanced nasopharyngeal carcinoma in taiwan," Frontiers in Pharmacology, vol. 11, Article ID 607413, 2020.

[5] L. Wang, W. Chen, M. Li, F. Zhang, K. Chen, and W. Chen, "A review of the ethnopharmacology, phytochemistry, pharmacology, and quality control of Scutellaria barbata D. Don," Journal of Ethnopharmacology, vol. 254, Article ID 112260, 2020.

[6] G. G. L. Yue, Y. Y. Chan, W. Liu et al., "Effectiveness of Scutellaria barbata water extract on inhibiting colon tumor growth and metastasis in tumor-bearing mice," Phytotherapy Research, vol. 35, no. 1, pp. 361-373, 2021.

[7] M. Wang, C. Ma, Y. Chen, X. Li, and J. Chen, "Cytotoxic neoclerodane diterpenoids fromScutellaria barbataD.Don," Chemistry and Biodiversity, vol. 16, no. 2, Article ID e1800499, 2019.

[8] L. Wang, J. Xu, Y. Yan, H. Liu, T. Karunakaran, and F. Li, "Green synthesis of gold nanoparticles from Scutellaria barbata and its anticancer activity in pancreatic cancer cell
(PANC-1)," Artificial Cells, Nanomedicine, and Biotechnology, vol. 47, no. 1, pp. 1617-1627, 2019.

[9] F. Guo, F. Yang, and Y.-H. Zhu, "Scutellarein from Scutellaria barbata induces apoptosis of human colon cancer HCT116 cells through the ROS-mediated mitochondria-dependent pathway," Natural Product Research, vol. 33, no. 16, pp. 2372-2375, 2019.

[10] X. Zheng, W. Kang, H. Liu, and S. Guo, "Inhibition effects of total flavonoids from Sculellaria barbata D. Don on human breast carcinoma bone metastasis via downregulating PTHrP pathway," International Journal of Molecular Medicine, vol. 41, no. 6, pp. 3137-3146, 2018.

[11] J. Liu, M. Jiang, Z. Li et al., "A novel systems pharmacology method to investigate molecular mechanisms of Scutellaria barbata D. Don for non-small cell lung cancer," Frontiers in Pharmacology, vol. 9, p. 1473, 2018.

[12] S. Li and B. Zhang, "Traditional Chinese medicine network pharmacology: theory, methodology and application," Chinese Journal of Natural Medicines, vol. 11, no. 2, pp. 110-120, 2013.

[13] L. Lu, S. Zhan, X. Liu, X. Zhao, X. Lin, and H. Xu, “Antitumor effects and the compatibility mechanisms of herb pair scleromitrion diffusum (Willd.) R. J. Wang-sculellaria barbata D. Don," Frontiers in Pharmacology, vol. 11, p. 292, 2020.

[14] Y. Yang, T. Fang, Y. L. Cao, Y. X. Lv, Q. Q. Chang, and D. D. Zhang, "Ethyl acetate fraction from hedyotis diffusa plus Scutellaria barbata exerts anti-breast cancer effect via miR200c-PDE7B/PD-L1-AKT/MAPK Axis," Evidence-based Complementary and Alternative Medicine: eCAM, vol. 2020, Article ID 3587095, 9 pages, 2020.

[15] X. Wu, S. Cui, Y. Wang, and R. Xu, "Research progress on anti-tumor mechanism of active ingredients of Chinese herbal medicine Scutellaria barbata," China Journal of Traditional Chinese Medicine and Pharmacy, vol. 33, no. 4, pp. 1459-1462, 2018.

[16] J. Dong, Y. Jin, and Y. Cao, "New advances in baicalein's antitumor effects and mechanisms," Journal of Pharmaceutical Practice, vol. 39, no. 1, pp. 9-12+43, 2021.

[17] W. Xiao, P. Bu, and W. Gong, "Research progress on antitumor and immunomodulatory effects of wogonin," China Journal of Chinese Materia Medica, vol. 39, no. 16, pp. 3004-3009, 2014.

[18] S. Jones and J. Z. Rappoport, "Interdependent epidermal growth factor receptor signalling and trafficking," The International Journal of Biochemistry \& Cell Biology, vol. 51, pp. 23-28, 2014.

[19] Z. A. Binder, A. H. Thorne, S. Bakas et al., "Epidermal growth factor receptor extracellular domain mutations in glioblastoma present opportunities for clinical imaging and therapeutic development," Cancer Cell, vol. 34, no. 1, pp. 163-177, 2018.

[20] L. Wulandari, G. Soegiarto, A. Febriani, F. Fatmawati, and Sahrun, "Comparison of detection of epidermal growth factor receptor (EFGR) gene mutation in peripheral blood plasma (liquid biopsy) with cytological specimens in lung adenocarcinoma patients," Indian journal of surgical oncology, vol. 12, no. Suppl 1, pp. 65-71, 2021.

[21] S. Cai, Y.-X. Zhang, K. Han, and Y.-Q. Ding, "Expressions and clinical significance of COX-2, VEGF-C, and EFGR in endometrial carcinoma," Archives of Gynecology and Obstetrics, vol. 296, no. 1, pp. 93-98, 2017.

[22] R. Liu, J. Liu, P. Wu, H. Yi, B. Zhang, and W. Huang, "Flotillin-2 promotes cell proliferation via activating the c-Myc/BCAT1 axis by suppressing miR-33b-5p in nasopharyngeal carcinoma," Aging, vol. 13, no. 6, pp. 8078-8094, 2021. 
[23] X. Yu, H. Li, P. Hu et al., "Natural HDAC-1/8 inhibitor baicalein exerts therapeutic effect in CBF-AML," Clinical and Translational Medicine, vol. 10, no. 4, p. e154, 2020.

[24] J. Wang, J. Li, Y. Wang, and Y. Zhu, "Baicalein induces apoptosis of bladder cancer cells via inhibition of the PI3K/ AKT/mTOR pathway," Journal of Shandong University(Health Sciences), vol. 57, no. 9, pp. 74-82, 2019.

[25] H. Tan, X. Li, W. H. Yang, and Y. Kang, "A flavone, Wogonin from Scutellaria baicalensis inhibits the proliferation of human colorectal cancer cells by inducing of autophagy, apoptosis and G2/M cell cycle arrest via modulating the PI3K/ AKT and STAT3 signalling pathways," Journal of B.U.ON: Official Journal of the Balkan Union of Oncology, vol. 24, no. 3, pp. 1143-1149, 2019. 